\title{
Indagine sulla diffusione di stafillococchi resistenti alla meticillina in Liguria
}

\begin{abstract}
Elisabetta Maioli, Roberto Bandettini, Enrico Battolla, Rosalba Bona, Luigi Carlo Bottaro, Rinaldo Brunetti, Roberto Capuzzo, Gian Luigi Devoto, Maria Dono, Andrea Dusi, Manuela Fedele, Giorgia Ferro, Giovanni Rosario Giusto, Stefania Mannelli, Federica Massucco, Maria Gabriella Mazzarello, Marco Mori, Mascja Perfumo, Gabriella Piatti, Silvia Reali, Agostina Ronca, Luisa Santoriello, Domizio Serra, David Usiglio, Anna Marchese, Eugenio A. Debbia
\end{abstract}

Gruppo Ligure sulla meticillino-resistenza, Sezione di Microbiologia C.A. Romanzi - DISCAT, Università di Genova

Prevalence of methicillin-resistant Staphylococci in a region of northern Italy (Liguria)

\section{SUMMARY}

Introduction. Staphylococcus aureus is a major opportunistic pathogen especially in nosocomial settings. An increasing proportion of strains has intrinsic resistance to methicillin (MRSA) and recent reports documented the emergence of MRSA also in the community. The aim of this study was to evaluate methicillin-resistance in Liguria.

Methods. 767 isolates of Staphylococcus spp have been collected from 10 laboratories, including 508 S. aureus: 292 (57.5\%) hospital-acquired, I 88 (37\%) community-acquired, and 28 (5.5\%) from healthcare settings; 259 coagulase-negative staphylococci (CNS): (193 (74.5\%) hospital-acquired, 56 (2I.6\%) community-acquired, and 10 (3.9\%) healthcare settings. Susceptibility tests were carried out by the disk diffusion method (CLSI, 2006) using cefoxitin (CFX) (Oxoid, Milan). MRSA has been also evaluated by PBP2' latex agglutination test (Oxoid, Milan) on 52 randomly selected $S$. aureus.

Results. The data obtained indicated an incidence of MRSA in nosocomial settings up to $45.2 \%$, while among the community-acquired isolates the percentage was $6.4 \%$. With respect to CNS the figures were $61,8 \%$ and $10,6 \%$ for the nosocomial-and community-acquired organisms respectively. A $99 \%$ agreement between data of the coordinating centre and the various laboratories was found. Methicillin-resistance was confirmed in $52 \mathrm{~S}$. aureus by PBP2' latex agglutination test.

Conclusions. Present findings indicate that MRSA in community in the Ligurian area exists and this phenomenon requires future surveillance.

\section{INTRODUZIONE}

Gli stafilococchi sono largamente diffusi in natura e sono tra i più importanti commensali nell'uomo ritrovandosi frequentemente sulla cute e sulle mucose dei tratti respiratorio e gastrointestinale. La specie più importante in patologia è sicuramente Staphylococcus aureus $(4,16)$. Poiché questo germe è in grado di elaborare una notevole quantità di enzimi che gli consentono di colonizzare gli ambienti più disparati (3), non sorprende di ritrovarlo in ambiente ospedaliero, dove rappresenta un microrganismo tra $i$ più frequentemente isolati nei reparti di terapia intensiva $(4,8,16)$. Tra gli stafilococchi coagulasi negativi di particolare interesse clinico sono: $S$. epidermidis, coinvolto in infezioni associate all'uso di cateteri sintetici e corpi estranei quali protesi valvolari cardiache, bypass atrioventricolari e protesi dell'anca, $S$. haemolyticus, associato a diverse infezioni quali endocardite, setticemia, peritonite, infezioni delle vie urinarie e $S$. saprophyticus, causa di infezioni alle vie urinarie in donne sessualmente attive. Altre specie di stafilococchi coagulasi negativi quali $S$. hominis, $S$. warneri, S. capitis, S. simulans, S. cohnii, S. xylosus, S. lugdunensis, S. schlefreri si incontrano molto più sporadicamente in campioni clinici $(2,8)$. Esistono diverse problematiche relative al trattamento delle infezioni da stafilococchi, dovute al fenomeno dell'acquisizione di resistenza agli antibiotici; all'epoca dell'introduzione della penicillina in terapia, infatti, gli stafilococchi risultavano sensibili a questa molecola, ma dopo soli 5 anni il 50\% dei microrganismi saggiati era diventato resistente grazie all'acquisizione di un plasmide che codifica la produzione di penicillinasi (19). La disponibilità di meticillina, una penicillina sintetica resistente alle ß-lattamasi, sembrò aprire un nuovo capitolo nella terapia antibiotica, tuttavia, entro un anno, vennero descritti i primi microorganismi resistenti a questo principio attivo e, a partire dagli anni '80 il numero delle segnalazioni riguardanti stafilococ- 
chi meticillino-resistenti (MRS) è aumentato in tutto il mondo (13). Inizialmente, le infezioni sostenute da stafilococchi meticillino-resistenti sono state riscontrate esclusivamente in ospedali universitari, ma gradualmente tali germi hanno cominciato a diffondersi anche ad altre strutture nosocomiali non universitarie $(11,19)$.

Attualmente è possibile riscontrare infezioni sostenute da MRS non solo nei reparti a rischio di grandi nosocomi ma anche in strutture più piccole, nei reparti day-hospital, nelle case di cura e, in alcuni rari casi, anche in ambiente comunitario $(7,14,15$, 22). Questo carattere ben radicato a livello ospedaliero in Italia, ma anche in altri Paesi (10) e che si accompagna a insensibilità anche ad altre classi di antibiotici, quali fluorochinoloni, macrolidi, aminoglicosidi, rende estremamente difficile il trattamento delle infezioni sostenute da tali germi (1719). In tale contesto il controllo della diffusione di questi microrganismi, sia in ambiente nosocomiale che in ambiente comunitario, appare fondamentale. Per questo motivo sono state attivate da più parti indagini per meglio valutare la proporzione del fenomeno e il quadro complessivo delle resistenze veicolate da questi patogeni.

Lo scopo di questo studio è stato quello di fotografare la situazione ligure valutando la distribuzione di Staphylococcus spp resistenti alla meticillina isolati da materiali patologici nei vari laboratori di Microbiologia Clinica in Liguria. Un preliminare resoconto di questo studio è stato presentato al XXXV Congresso Nazionale AMCLI, Torino, 2006.

\section{MATERIALI E METODI}

Nel periodo tra febbraio e maggio 2006, è stato richiesto a vari laboratori di Microbiologia Clinica del territorio ligure di collezionare 100 ceppi di stafilococchi isolati consecutivamente da pazienti diversi. Il protocollo prevedeva inoltre la raccolta del maggior numero di informazioni clinico demografiche. Non tutti i ceppi forniti hanno potuto essere inclusi nello studio perché non raccolti secondo i criteri stabiliti dal protocollo. Sono stati collezionati 767 ceppi di Staphylococcus spp da 10 centri liguri, così suddivisi: 508 S. aureus, 259 Staphylococcus coagulasi-negativi (SCN) (150 S. epidermidis, 37 S. haemoliticus, 28 S. hominis, 8 S. saprofiticus, $7 \mathrm{~S}$. capitis, $5 \mathrm{~S}$. simulans, $5 \mathrm{~S}$. warnerii, 3 S. cohnii, 3 S. spp., 3 S. intermedius, 2 S. lugdunensis, 2 S. schlefreri, $2 S$. sciuri, 2 S. xylosus, 1 S. caprae, 1 S. lentus). Dei 508 S. aureus, 292 erano di provenienza nosocomiale $(57.5 \%), 188$ $(37 \%)$ di origine comunitaria mentre $28(5.5 \%)$ erano di origine comunitaria con fattore di rischio (stipiti isolati da pazienti provenienti da residenze protette o sottoposti a dialisi); dei 259 stafilococchi coagulasi negativi raccolti, $193(74.5 \%)$ erano di provenienza nosocomiale, $56(21.6 \%)$ di origine comunitaria mentre $10(3.9 \%)$ erano di origine comunitaria con fattore di rischio.

Dopo averne valutato la corretta identificazione mediante test della coagulasi e semina su piastre al mannitolo, i ceppi inviati al laboratorio sono stati analizzati al fine di determinarne la meticillino-resistenza con la metodica suggerita da CLSI (2006) mediante la tecnica di diffusione da dischetto e utilizzando come paragone la cefoxitina come riportato altrove $(1,5)$. È stata inoltre valutata la produzione di PBP2' su 52 ceppi campione di $S$. aureus scelti a caso tra quelli inclusi nello studio mediante il test di agglutinazione al lattice al fine di rilevare ceppi MRSA. I dati complessivi così ottenuti sono stati poi elaborati e confrontati con i risultati ottenuti dai laboratori centri di origine degli stipiti. Hanno partecipato allo studio i seguenti centri: Centro 1, ASL 1 Imperiese, Imperia; Centro 2, ASL2 Ospedale San Paolo, Savona; Centro 3 ASL 3 San Carlo, Genova-Voltri; Centro 4, Azienda Ospedaliera "Villa Scassi", Genova-Sampierdarena; Centro 5, Ente Ospedaliero "Ospedali Galliera", Genova; Centro 6, Ospedale Evangelico Internazionale, Genova; Centro 7, UO di Microbiologia Clinica, sez. Microbiologia Università degli Studi di Genova; Centro 8, Istituto Giannina Gaslini, Genova; Centro 9, ASL 4 Chiavarese, Genova; Centro 10, ASL 5 Spezzina, Ospedale Civile S. Andrea, La Spezia.

\section{RISULTATI}

Dai risultati ottenuti utilizzando la resistenza alla cefoxitina come indice di meticillino-resistenza, è emerso che dei 767 ceppi saggiati, 352 (45.9\%) sono risultati cefoxitino-resistenti (tabella 1).

Tabella I. Percentuali di resistenza a cefoxitina dei 767 ceppi raccolti durante lo studio

\begin{tabular}{|c|c|c|}
\hline & $\mathbf{N}^{\circ}$ CEPPI (\%) & CEFOXITINA-R (\%) \\
\hline Staphylococcus spp & 767 & $352(45.9 \%)$ \\
\hline Nosocomiali & $485(63.2 \%)$ & $300(61.8 \%)$ \\
\hline Comunitari & $244(31.8 \%)$ & $26(10.6 \%)$ \\
\hline $\begin{array}{l}\text { Comunitari con } \\
\text { fattore di rischio }\end{array}$ & $38(5 \%)$ & $26(68.4 \%)$ \\
\hline S. aureus & $508(66.2 \%)$ & $161(31.7 \%)$ \\
\hline Nosocomiali & $292(57.5 \%)$ & $132(45.2 \%)$ \\
\hline Comunitari & $188(37 \%)$ & $12(6.4 \%)$ \\
\hline $\begin{array}{l}\text { Comunitari con } \\
\text { fattore di rischio }\end{array}$ & $28(5.5 \%)$ & $17(60.7 \%)$ \\
\hline S. coag. neg. & $259(33.8 \%)$ & $191(73.7 \%)$ \\
\hline Nosocomiali & $193(74.5 \%)$ & $168(87 \%)$ \\
\hline Comunitari & $56(21.6 \%)$ & $14(25 \%)$ \\
\hline $\begin{array}{l}\text { Comunitari con } \\
\text { fattore di rischio }\end{array}$ & $10(3.9 \%)$ & $9(90 \%)$ \\
\hline
\end{tabular}


Per quanto riguarda $S$. aureus, sono stati osservati $161(31.7 \%)$ ceppi resistenti. Il confronto con i dati ottenuti dai centri di origine dei ceppi ha mostrato che dei 508 isolati di $S$. aureus giunti al centro di riferimento, 505 ceppi (99\%), hanno confermato la sensibilità o la resistenza alla cefoxitina come riportato nel laboratorio d'origine.

Tra gli stafilococchi coagulasi negativi, 191 (73.7\%) hanno evidenziato oxacillino-resistenza. La concordanza tra il dato ottenuto nel centro partecipante e quello di riferimento è stata del $97.3 \%$ (tabella 1).

La situazione dei singoli centri è riportata in dettaglio nelle tabelle 2-11.

Tabella 2. Percentuali di resistenza a cefoxitina dei ceppi raccolti presso il Centro I.

\begin{tabular}{lcc}
\hline & $N^{\circ}$ CEPPI (\%) & CEFOXITINA-R (\%) \\
\hline Staphylococcus spP & III & $46(4 I .4 \%)$ \\
\hline Nosocomiali & $73(65.8 \%)$ & $40(54.8 \%)$ \\
\hline Comunitari & $29(26.1 \%)$ & - \\
\hline $\begin{array}{l}\text { Comunitari con } \\
\text { fattore di rischio }\end{array}$ & $9(8.1 \%)$ & $6(66.6 \%)$ \\
\hline & & \\
\hline S. aureus & $65(58.6 \%)$ & $15(23.1 \%)$ \\
\hline Nosocomiali & $38(58.5 \%)$ & $13(34.2 \%)$ \\
\hline Comunitari & $23(35.4 \%)$ & - \\
\hline $\begin{array}{l}\text { Comunitari con } \\
\text { fattore di rischio }\end{array}$ & $4(6.1 \%)$ & $2(50 \%)$ \\
\hline & & $31(67.4 \%)$ \\
\hline S. coag. neg. & $46(41.4 \%)$ & $27(77.1 \%)$ \\
\hline Nosocomiali & $35(76.1 \%)$ & - \\
\hline Comunitari & $6(13 \%)$ & $4(80 \%)$ \\
\hline Comunitari con & & \\
fattore di rischio & $5(10.9 \%)$ & \\
\hline
\end{tabular}

Tabella 3. Percentuali di resistenza a cefoxitina dei ceppi raccolti presso il Centro 2

\begin{tabular}{|c|c|c|}
\hline & $\mathbf{N}^{\circ}$ CEPPI (\%) & CEFOXITINA-R (\%) \\
\hline Staphyococcus spp & $4 I$ & $21(5 I .2 \%)$ \\
\hline Nosocomiali & $32(78.1 \%)$ & $20(62.5 \%)$ \\
\hline Comunitari & $9(21.9$ & $\mathrm{I}(\mathrm{II} . \mathrm{I} \%)$ \\
\hline \multicolumn{3}{|l|}{ Comunitari con } \\
\hline fattore di rischio & - & - \\
\hline S. aureus & $28(68.3 \%)$ & II(39.3\%) \\
\hline Nosocomiali & $20(71.4 \%)$ & $10(50 \%)$ \\
\hline Comunitari & $8(28.6 \%)$ & $\mathrm{I}(\mathrm{I} 2.5 \%)$ \\
\hline \multicolumn{3}{|l|}{ Comunitari con } \\
\hline fattore di rischio & - & - \\
\hline S. coag. neg. & $13(31.7 \%)$ & $10(76.9 \%)$ \\
\hline Nosocomiali & $12(92.3 \%)$ & $10(83.3 \%)$ \\
\hline Comunitari & $\mathrm{I}(7.7 \%)$ & - \\
\hline Comunitari con & _ & - \\
\hline
\end{tabular}

Tabella 4. Percentuali di resistenza a cefoxitina dei ceppi raccolti presso il Centro 3

\begin{tabular}{lcc}
\hline & $\mathbf{N}^{\circ}$ CEPPI (\%) & CEFOXITINA-R (\%) \\
\hline Staphylococcus spP & I 20 & $59(49.2 \%)$ \\
\hline Nosocomiali & $75(62.5 \%)$ & $51(68 \%)$ \\
\hline Comunitari & $45(37.5 \%)$ & $8(17.8 \%)$ \\
\hline Comunitari con & & - \\
fattore di rischio & - & - \\
\hline
\end{tabular}

\begin{tabular}{|c|c|c|}
\hline S. aureus & $7 I(59.2 \%)$ & $28(39.4 \%)$ \\
\hline Nosocomiali & $49(69 \%)$ & $25(51 \%)$ \\
\hline Comunitari & $22(31 \%)$ & $3(13.6 \%)$ \\
\hline $\begin{array}{l}\text { Comunitari con } \\
\text { fattore di rischio }\end{array}$ & - & - \\
\hline S. coag. neg. & $49(40.8 \%)$ & $3 I(63.3 \%)$ \\
\hline Nosocomiali & $26(53.1 \%)$ & $26(100 \%)$ \\
\hline Comunitari & $23(46.9 \%)$ & $5(21.7 \%)$ \\
\hline $\begin{array}{l}\text { Comunitari con } \\
\text { fattore di rischio }\end{array}$ & - & - \\
\hline
\end{tabular}

Tabella 5. Percentuali di resistenza a cefoxitina dei ceppi raccolti presso il Centro 4

\begin{tabular}{|c|c|c|}
\hline & $\mathbf{N}^{\circ}$ CEPPI (\%) & CEFOXITINA-R (\%) \\
\hline Staphylococcus spp & 20 & $15(75 \%)$ \\
\hline Nosocomiali & $17(85 \%)$ & $14(82.3 \%)$ \\
\hline Comunitari & $3(15 \%)$ & $\mathrm{I}(33.3 \%)$ \\
\hline $\begin{array}{l}\text { Comunitari con } \\
\text { fattore di rischio }\end{array}$ & - & - \\
\hline S. aureus & $7(35 \%)$ & $3(42.8 \%)$ \\
\hline Nosocomiali & $5(7 \mid .4 \%)$ & $3(60 \%)$ \\
\hline Comunitari & $2(28.6 \%)$ & - \\
\hline $\begin{array}{l}\text { Comunitari con } \\
\text { fattore di rischio }\end{array}$ & - & - \\
\hline S. coag. neg. & $13(65 \%)$ & $12(92.3 \%)$ \\
\hline Nosocomiali & $12(92.3 \%)$ & $\mathrm{II}(9 \mathrm{I} .7 \%)$ \\
\hline Comunitari & $\mathrm{I}(7.7 \%)$ & $\mathrm{I}(\mathrm{I} 00 \%)$ \\
\hline $\begin{array}{l}\text { Comunitari con } \\
\text { fattore di rischio }\end{array}$ & - & - \\
\hline
\end{tabular}

Tabella 6. Percentuali di resistenza a cefoxitina dei ceppi raccolti presso il Centro 5

\begin{tabular}{|c|c|c|}
\hline & $\mathbf{N}^{\circ}$ CEPPI (\%) & CEFOXITINA-R (\%) \\
\hline Staphylococcus spp. & 49 & $23(46.9 \%)$ \\
\hline Nosocomiali & $20(40.8 \%)$ & $13(5 \%)$ \\
\hline Comunitari & $23(46.9 \%)$ & $5(21.7 \%)$ \\
\hline $\begin{array}{l}\text { Comunitari con } \\
\text { fattore di rischio }\end{array}$ & $6(12.3 \%)$ & $5(83.3 \%)$ \\
\hline S.aureus & $25(51 \%)$ & $9(36 \%)$ \\
\hline Nosocomiali & $9(36 \%)$ & $4(44.4 \%)$ \\
\hline Comunitari & $14(56 \%)$ & $3(21.4 \%)$ \\
\hline $\begin{array}{l}\text { Comunitari con } \\
\text { fattore di rischio }\end{array}$ & $2(8 \%)$ & $2(100 \%)$ \\
\hline S. coag. neg. & $24(49 \%)$ & $14(58.3 \%)$ \\
\hline Nosocomiali & $\mathrm{II}(45.8 \%)$ & $9(81.8 \%)$ \\
\hline Comunitari & $9(37.5 \%)$ & $2(22.2 \%)$ \\
\hline $\begin{array}{l}\text { Comunitari con } \\
\text { fattore di rischio }\end{array}$ & $4(16.7 \%)$ & $3(75 \%)$ \\
\hline
\end{tabular}


Tabella 7. Percentuali di resistenza a cefoxitina dei ceppi raccolti presso il Centro 6

\begin{tabular}{|c|c|c|}
\hline & $\mathbf{N}^{\circ}$ CEPPI (\%) & CEFOXITINA-R (\%) \\
\hline Staphylococcus spp & 57 & $25(43.8 \%)$ \\
\hline Nosocomiali & $24(42.1 \%)$ & $14(58.3 \%)$ \\
\hline Comunitari & $17(29.8 \%)$ & $\mathrm{I}(5.9 \%)$ \\
\hline $\begin{array}{l}\text { Comunitari con } \\
\text { fattore di rischio }\end{array}$ & $16(28.1 \%)$ & $10(62.5 \%)$ \\
\hline S. aureus & $55(96.5 \%)$ & $23(41.8 \%)$ \\
\hline Nosocomiali & $23(4 I .8 \%)$ & $13(56.5 \%)$ \\
\hline Comunitari & $16(29.1 \%)$ & - \\
\hline $\begin{array}{l}\text { Comunitari con } \\
\text { fattore di rischio }\end{array}$ & $16(29.1 \%)$ & $10(62.5 \%)$ \\
\hline S. coag. neg. & $2(3.5 \%)$ & $2(100 \%)$ \\
\hline Nosocomiali & $\mathrm{I}(50 \%)$ & $I(100 \%)$ \\
\hline Comunitari & $I(50 \%)$ & $\mathrm{I}(100 \%)$ \\
\hline $\begin{array}{l}\text { Comunitari con } \\
\text { fattore di rischio }\end{array}$ & - & - \\
\hline
\end{tabular}

Tabella 8. Percentuali di resistenza a cefoxitina dei ceppi raccolti presso il Centro 7

$\mathbf{N}^{\circ}$ CEPPI (\%) CEFOXITINA-R (\%)

\begin{tabular}{lcc}
\hline Staphylococcus spp & 27 & $13(48.1 \%)$ \\
\hline Nosocomiali & $25(92.6 \%)$ & $13(52 \%)$ \\
\hline Comunitari & $2(7.4 \%)$ & - \\
\hline Comunitari con & & - \\
fattore di rischio & - & \\
\hline
\end{tabular}

\begin{tabular}{lcc}
\hline S. aureus & $20(74.1 \%)$ & $9(45 \%)$ \\
\hline Nosocomiali & $18(90 \%)$ & $9(50 \%)$ \\
\hline Comunitari & $2(10 \%)$ & - \\
\hline Comunitari con & & - \\
fattore di rischio & - & - \\
\hline
\end{tabular}

\begin{tabular}{lcc}
\hline S. coag. neg. & $7(25.9 \%)$ & $4(57.1 \%)$ \\
\hline Nosocomiali & $7(100 \%)$ & $4(57.1 \%)$ \\
\hline Comunitari & - & - \\
\hline Comunitari con & & - \\
fattore di rischio & - & - \\
\hline
\end{tabular}

Tabella 9. Percentuali di resistenza a cefoxitina dei ceppi raccolti presso il Centro 8

\begin{tabular}{|c|c|c|}
\hline - & $\mathbf{N}^{\circ}$ CEPPI (\%) & CEFOXITINA-R (\%) \\
\hline Staphylococcus spp & 22 & $9(40.9 \%)$ \\
\hline Nosocomiali & $21(95.5 \%)$ & $9(42.8 \%)$ \\
\hline Comunitari & $\mathrm{I}(4.5 \%)$ & - \\
\hline $\begin{array}{l}\text { Comunitari con } \\
\text { fattore di rischio }\end{array}$ & - & - \\
\hline S. aureus & $12(54.5 \%)$ & $2(16.7 \%)$ \\
\hline Nosocomiali & $\mathrm{II}(9 \mathrm{I} .7 \%)$ & $2(18.2 \%)$ \\
\hline Comunitari & $\mathrm{I}(8.3 \%)$ & - \\
\hline $\begin{array}{l}\text { Comunitari con } \\
\text { fattore di rischio }\end{array}$ & - & - \\
\hline
\end{tabular}

\begin{tabular}{lcc}
\hline S. coag. neg. & $10(100 \%)$ & $7(70 \%)$ \\
\hline Nosocomiali & $10(100 \%)$ & $7(70 \%)$ \\
\hline Comunitari & - & - \\
\hline $\begin{array}{l}\text { Comunitari con } \\
\text { fattore di rischio }\end{array}$ & - & - \\
\hline
\end{tabular}

Tabella 10. Percentuali di resistenza a cefoxitina dei ceppi raccolti presso il Centro 9

\begin{tabular}{|c|c|c|}
\hline & $\mathbf{N}^{\circ}$ CEPPI (\%) & CEFOXITINA-R (\%) \\
\hline Staphylococcus spp & 150 & $93(62 \%)$ \\
\hline Nosocomiali & $102(68 \%)$ & $84(82.3 \%)$ \\
\hline Comunitari & 47 (3I.3\%) & $8(17 \%)$ \\
\hline $\begin{array}{l}\text { Comunitari con } \\
\text { fattore di rischio }\end{array}$ & I (0.7\%) & I (100\%) \\
\hline S. aureus & $95(63.3 \%)$ & $43(45.3 \%)$ \\
\hline Nosocomiali & $55(57.9 \%)$ & $38(69.1 \%)$ \\
\hline Comunitari & $40(42.1 \%)$ & $5(12.5 \%)$ \\
\hline $\begin{array}{l}\text { Comunitari con } \\
\text { fattore di rischio }\end{array}$ & - & - \\
\hline S. coag. neg. & $55(36.7 \%)$ & $50(53.8 \%)$ \\
\hline Nosocomiali & 47 (85.5\%) & $46(97.9 \%)$ \\
\hline Comunitari & $7(12.7 \%)$ & $3(42.8 \%)$ \\
\hline $\begin{array}{l}\text { Comunitari con } \\
\text { fattore di rischio }\end{array}$ & I (I.8\%) & I (100\%) \\
\hline
\end{tabular}

Tabella I I. Percentuali di resistenza a cefoxitina dei ceppi raccolti presso il Centro 10

\section{N CEPPI (\%) CEFOXITINA-R (\%)}

\begin{tabular}{lcc}
\hline Staphylococcus spp & 170 & $46(27 \%)$ \\
\hline Nosocomiali & $96(56.5 \%)$ & $4 \mathrm{I}(42.7 \%)$ \\
\hline Comunitari & $68(40 \%)$ & $3(4.4 \%)$ \\
\hline $\begin{array}{l}\text { Comunitari con } \\
\text { fattore di rischio }\end{array}$ & $6(3.5 \%)$ & $2(33.3 \%)$ \\
\hline & & \\
\hline S. aureus & $130(76.5 \%)$ & $17(13.1 \%)$ \\
\hline Nosocomiali & $64(49.2 \%)$ & $15(23.4 \%)$ \\
\hline Comunitari & $60(46.2 \%)$ & - \\
\hline $\begin{array}{l}\text { Comunitari con } \\
\text { fattore di rischio }\end{array}$ & $6(4.6 \%)$ & $2(33.3 \%)$ \\
\hline & & $29(72.5 \%)$ \\
\hline $\begin{array}{l}\text { S. coag. neg. } \\
\text { Nosocomiali }\end{array}$ & $32(23.5 \%)$ & $26(81.2 \%)$ \\
\hline Comunitari & $8(4.7 \%)$ & $3(37.5 \%)$ \\
\hline Comunitari con & & - \\
fattore di rischio & - & \\
\hline
\end{tabular}

Come atteso la diffusione della resistenza alla meticillina è molto variabile in funzione dell'area geografica considerata e dell'origine dei ceppi nosocomiali o comunitari. In particolare le percentuali di MRSA e SCN meticillino-resistenti variavano rispettivamente dal 18.2 al $69.1 \%$ e dal 70 al $100 \%$.

In comunità, considerando i centri che sono riusciti a fornire almeno 10 stipiti, le percentuali di meticillino-resistenza oscillavano tra lo $0 \%$ sino ad un massimo del $20 \%$ sia per S. aureus che per SCN. Ceppi comunitari con fattori di rischio sono stati forniti solo da un numero limitato di centri: 4 e 3 rispettivamente per $S$. aureus e SCN. Per questi ultimi microrganismi, come atteso l'incidenza di meticillino-resistenza era più elevata rispetto ai ceppi comunitari senza fattori di 
rischio: 57 e $70 \%$ sul totale degli stafilococchi raccolti nello studio.

Il test di agglutinazione al lattice per la rilevazione della PBP2' in $S$. aureus ha identificato i ceppi MRSA nel $100 \%$ dei casi se confrontati con i risultati ottenuti con la tradizionale tecnica di diffusione da dischetto di cefoxitina.

\section{CONCLUSIONI}

Il primo caso di stafilococco meticillino-resistente venne riportato nel 1961, un anno dopo l'introduzione di questa penicillina semi-sintetica (9). Da allora tale fenomeno non si è mai arrestato interessando, con il tempo e in diversa misura, $\mathrm{i}$ nosocomi di vari paesi. Di particolare interesse risulta la percentuale della meticillino-resistenza in $S$. aureus riscontrata nelle nazioni europee: essa varia di circa 100 volte, da valori inferiori all'1\% dei paesi del nord Europa, a valori del $40 \%$ e oltre di Grecia, Regno Unito e Italia (20-21). Questo studio ha dimostrato che, in linea con quanto sopra citato, anche nei nosocomi liguri la meticillino-resistenza è diffusa con valori di poco al di sopra di quelli registrati in ambito nazionale. È da sottolineare il fatto che mentre inizialmente gli stafilococchi meticillino-resistenti si riscontravano unicamente in ambito ospedaliero, attualmente si cominciano a rinvenire anche in ambito comunitario, un evento facilmente prevedibile. Nell'area ligure il fenomeno appare incidere per circa un $7 \%$ in $S$. aureus e in maggior misura tra $i$ ceppi CNS. Tali dati necessitano di ulteriori conferme anche con studi più approfonditi per meglio identificare il genotipo degli isolati. Tutti i germi isolati in comunità sono risultati totalmente sensibili alla clindamicina, come riportato altrove (12) e alla rifampicina, ma non sono state osservate particolari differenze di sensibilità alle altre classi di antibiotici tra i ceppi comunitari e quelli nosocomiali. Il presente studio indica che ormai la diffusione di questi cloni seppur ancora limitata è iniziata e sarà destinata a consolidarsi, come tutte le evoluzioni verso la resistenza agli antibiotici che abbiamo osservato negli anni recenti, se fattori diversi non interverranno a circoscriverne la portata.

\section{BIBLIOGRAFIA}

1. Andrews J, Brenwald N, Brown DFJ, Perry J, King A, Gemmel C. Evaluation of a $10 \mu \mathrm{g}$ cefoxitin disc for the detection of methicillin resistance in Staphylococcus aureus by BSAC methodology. J Antimicrob Chemoter 2005; 267: 599-600.

2. Archer GL, Climo MW. Staphylococcus epidermidis and other coagulase-negative staphylococci. In Mandell GL, Bennet JE, Dolin R. Principles and Practice of Infectious Disease. Sixth Ed. Churchill Livingstone Philadelphia, USA 2005; 2352-60.
3. Archer GL. Staphylococcus aureus: a well armed pathogen. Clin Infect Dis 1998; 26: 1179-81.

4. Bannerman TL. Staphylococcus, Micrococcus and other catalase positive cocci that grow aerobically. In Murray PR, Baron EJ, Jorgensen JH, Pfaller MA, Yolken RH, eds. Washington, DC: American Society for Microbiology Press. Manual of Clinical Microbiology 2003; 8th ed.: 384-404.

5. Brown DFJ, Edwards DI, Hawkey PM, et al. Guidelines for the laboratory diagnosis and susceptibility testing of methicillin-resistant Staphylococcus aureus (MRSA). J Antimicrob Chemoter 2005; 56: 1000-18.

6. Clinical and Laboratory Standards Institute. Performance Standard for Antimicrobial Susceptibility Tests; Fifteenth Informational Supplement. M2-A8. and Supplement M100-S16, Wayne, PA, 2006.

7. Cooper BS, Medley GF, Stone SP, et al. Methicillinresistant Staphylococcus aureus in hospitals and the community: Stealth dynamics and control catastrophes. Proc Natl Acad Sci USA 2004; 101: 10223-8.

8. Foster TJ. Immune evasion by staphylococci Nat Rev Microbiol 2005; 3: 948-58.

9. Jevons MP. Celbenin-resistant staphylococci Br Med J 1961; i: 124-5.

10. Jones ME, Karlowsky JA, Draghi DC, Thornsberry C, Sahm DF, Bradley JS. Rates of antimicrobial resistance among common bacterial pathogens causing respiratory, blood, urine, and skin and soft tissue infections in pediatric patients. Eur J Clin Microbiol Infect Dis 2004; 23: 445-55.

11. Klutymans-VandenBergh M, Kltymans J. Community-acquired MRSA: current perspectives. Clin Microbiol Infect 2006; 12 (suppl. 1): 9-15.

12. Liao RS, Storch GA, Buller RS, et al. Blinded comparaison of repetitive-sequence PCR and multilocus sequence typing for genotyping methicillin-resistant Staphylococcus aureus isolates from a children's hospital in St. Louis, Missouri. J Clin Microbiol 2006; 44: 2254-7.

13. Maranan MC, Moreira B, Boyle-Vavra S, Daum RS. Antimicorbial resistance in staphylococci. Epidemiology, molecular mechanism and clinical relevance. Infect Dis Clin North Am 1997; 11: 81349.

14. Moellering RC Jr. The growing menace of community-acquired methicillin-resistant Staphylococcus aureus. Ann Inter Med 2006; 144: 368-70.

15. Mongkolrattanothai K, Boyle S, Kahana MD, Daum RS. Severe Staphylococcus aureus infections caused by clonally related community-acquired methicillinsusceptible and methicillin-resistant isolates. Clin Infect Dis 2003; 37: 1050-8.

16. Moreillon P, Que YA, Glauser MP. Staphylococcus aureus, including staphylococcal toxic shock. In Mandell GL, Bennet JE, Dolin R. Principles and Practice of Infectious Disease. Churchill Livingstone. Philadelphia, USA. Sixth Ed. 2005; 2321-51.

17. Raad I, Alrahwan A, Rolston K. Staphylococcus epidermidis: emerging resistance and need for alternative agents. Clin Inf Dis 1998; 26: 1182-7.

18. Robinson DA, Enright MC. Evolutionary models of the emergence of methicillin-resistant Staphylococcus aureus. Antimicrob Agents Chemoter 2003; 47: 392634

19. Schito GC. The importance of the development of antibiotic resistance in Staphylococcus aureus. Clin Microb and Infections 2006; 2 (suppl. 1). 
20. Stefani S, Varaldo PE. Epidemiology of methicillinresistant staphylococci in Europe. Clin Microbiol Infect 2003; 9: 1179-86.

21. Tiemersma EW, Bronzwaer SL, Lyytikainen O, et al. Methicillin-resistant Staphylococcus aureus in Europe, 1999-2000. Emerg Infect Dis 2004; 10: 1627 34.

20. Vandenesch F, Naimi T, Enright MC, et al. Community-acquired methicillin-resistant Staphylococcus aureus carrying Panton-Valentine leukocidin genes: worldwide emergence. Emer Infect Dis 2003; 9: 978-84.

Eugenio A. Debbia

Sez. Microbiologia C.A. Romanzi DISCAT, Università degli Studi di Genova Largo Rosanna Benzi, 10

16132 Genova

Tel.: + 39-10-3537655 Fax: +39-10-3537698

E-mail: eugenio.debbia@unige.it

http://www.microbiologia.unige.it/dpb/debbia.htm 